10 Altschul S F, Madden T L, Schaffer A, et al. Gappped BLAST and PST-BLAST: a new generation of protein database search programs. Nucleic Acid Res, 1997, 25: 3389 3402

11 Thompson J D, HIgins D G, Gibson T J. CLUSTAL W: improving the sensitivity of progressive multiple sequence alignment through sequence weighting, positions-specific gap penalties and eight matrix choice. Nucleic Acids Res, 1994, 22: 4673 4680

12 Kozak M. Recognition of AUG and alternative initiator codons is augmented by G in position +4 but is not generally affected by the nucleotides in positions +5 and +6 . The EMBO Journal, 1997, 16: 2482 2492

13 Jurata L W, Gill G N. The Functional analysis of the nuclear LIM domain interactor NLI. Molecular and Cell Biology, 1997, 17: 5688 5698

14 Bach I, Carriere C, Ostendorff H P, et al. A family of LIM domairr associated cofactors confers transcriptional synergism between LIM and Otx hoeodomain proteins. Genes \& Development, 1997, 11: 1370 1380

15 Wadman I A, Osada H, Grutz G G, et al. The LIM-only protein Lmo2 is a bridging molecule assembling an erythroid, DNA-binding complex which includes the TAL. 1, E47, GATA-1 and Ldb1/ NLI proteins. The EMBO Journal, 1997: 3145 3157

16 Morcillo P, Rosen C, Dorsett D. Genes regulating the remote wing margin enhancer in the Drosophila cut locus. Genetics, 1996, 144: 1143 1154

17 Agulnick A D, Taira M, Breen J J, et al. Interactions of the LIM-domairr binding factor Ldb1 with LIM homeodomain proteins. Nature, 1996, 384: 270 272

18 Visvader J E, Mao X, Fujiwara Y, et al. The LIM-domain binding protein Ldb1 and its partner LMO2 act as negative regulators of erythroid differentiation. Proc Natl Acad Sci USA, 1997, 94: 13707 13712

\title{
地球外核流体运动与磁场长期 变化相互关系的探讨
}

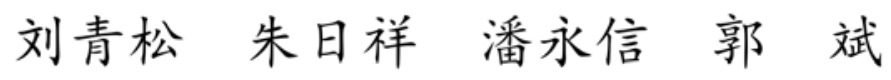

(中国科学院地球物理研究所, 北京 100101)

摘要从理论上分析了偶极子场的能谱分布特征, 在高阶项, 它近似为一直线, 斜率与偶极子 的深度相关. 地球磁场等效磁源深度 $\left(r_{0}\right)$ 并非一个常量: 1900 1940 年 $r 1$ 稳定递减; 1940 1950 年 $r_{0}$ 的阶跃性变化可能与某种扰动相关; 1950 1975 年 $r_{0}$ 呈现递减趋势; 1975 1985 年 稳定递增; 1985 1995 年 $r_{0}$ 则基本保持不变. 把每一年的能谱归一到相应的等效磁源深度, 发现 1900 1960 年偶极子场的能量基本保持不变, 非偶极子场的能量增加, 地球磁场总能量 逐年递增. 在此期间地表观测的磁场能量衰减是由等效磁源的深度变化引起. 忽略 1940 1950 年的扰动, $r_{0}$ 的变化在 1975 及 1985 年明显出现拐点. 这一变化规律与地磁阶跃 ( jerk) 及日长变化趋势一致, 但滞后于地磁阶跃 5 a. 初步研究表明, 核、幔耦合引起外核流体运动方 式的改变, 不但能够引起地磁阶跃及日长的变化, 而且还能引起 $r_{0}$ 的长期变化.

\section{关键词 等效磁源深度 地磁阶跃 日长}

对近一个世纪以来地球磁场的研究表明, 地球磁场 1969 年在世界范围内发生阶跃现象, 其时间尺度仅为年 ${ }^{[1]}$. 如果这一阶跃信号起源于核、幔边界, 那么会为反演下地幔的电导率提 供新的约束. 地磁阶跃与核、幔之间电磁耦合及日长变化有关 ${ }^{1]}$. 基于磁流体冻结近似 
(frozen-flux approximation) 及稳定流体假设( steady flow), 可以根据地表观测的地磁场反演外核 顶部流体的运动 ${ }^{[2 ~ 4]}$. 但是由于上述解所固有的非唯一性, 及在求解过程中稳定流体假设具 有的平滑性, 无法模拟地磁阶跃 ${ }^{[5]}$. 地球磁场长期变化与日长变化存在着相关性 ${ }^{[6]}$, 地球角 动量的改变有可能是地磁阶跃的起因 ${ }^{[7]}$, 但是地球角动量的变化趋势与地磁阶跃并不完全一 致.

本文将从地球磁场变化本身出发, 寻求地磁阶跃的起因. 借助于高斯系数 $g_{n}^{m}$ 和 $h_{n}^{m}$, 地球 磁场在地表的能谱可以表示为 ${ }^{[8]}$

$$
W(n)=(n+1) \sum_{m=0}^{n}\left(g_{n}^{m^{2}}+h_{n}^{m^{2}}\right) .
$$

在对数坐标下, $W(n)$ 的高阶部分 $(n=2, \cdots, 11,12)$ 近似为一直线 ${ }^{[9 \sim 11]}$, 其斜率可以用 来确定等效磁源到地心的距离 $r_{0}$. 从统计意义上讲, $r_{0}$ 代表产生地球磁场源的平均深度. Benton 等人 ${ }^{[1]}$ 的 $r_{0}$ 位于核、幔边界之下, 暗示 $r_{0}$ 变化应起因于外核. 在地表观测的偶极子分 量占地球磁场的 $85 \%$, 其磁矩在过去 $0.1 \mathrm{ka}$ 中降低了近 $5 \%$, 然而当把地球磁场在地表的能谱 归一到同一等效磁源深度时, 发现地球磁场偶极子的能量衰减, 而非偶极子的能量增加, 总能 谱基本保持守恒, 能量从偶极子场向非偶极子场转化 ${ }^{[12]}$. 可见地球磁场在地表及在地球深部 的特性是不一样的, $r_{0}$ 及其变化对于研究地球磁场时空结构具有重要意义. Meyer ${ }^{[12]}$ 把不同 年代地球磁场的能谱下延到同一参考面, 没有考虑 $r_{0}$ 随时间的变化及其对能谱分布的影响. 本文将对这一问题加以探讨, 同时将根据日长变化已有的研究成果, 探讨 $r_{0}$ 演化的机制, 并寻 求 jerk 的形成机制.

\section{1 地球磁场的能谱及等效磁源}

假定一个偶极子的位置在 $r_{0}, \theta_{0}$ 和 $\varphi_{0}$, 磁矩为 $M_{r}$. 其磁位可以表达为 ${ }^{[13]}$

$$
u_{r}=a \sum_{n=1}^{\infty} \sum_{m=0}^{n}\left|\frac{a}{r}\right|^{n+1}\left(g_{n}^{m} \cos (m \lambda)+h_{n}^{m} \sin (m \lambda)\right) P_{n}^{m}(\cos \theta),
$$

其中 $g_{n}^{m}=\left|\frac{M_{r}}{a^{3}}\right|\left|\frac{r_{0}}{a}\right|^{n-1} n P_{n}^{m}(\cos \theta) \cos \left(m \lambda_{0}\right), h_{n}^{m}=g_{n}^{m} \tan \left(m \lambda_{0}\right)$, 这一模型预测 $h_{n}^{m}$ 与 $g_{n}^{m}$ 具有 相同的变化率. 采用与 (1) 式相同的形式, 偶极子的理论能谱密度函数为

$$
W(n)=(n+1) n^{2}\left|\frac{M_{r}}{a^{3}}\right|\left|\frac{r_{0}}{a}\right|^{2[n-1]} \sum_{m=0}^{n} P_{n}^{m}\left(\cos \left(m \lambda_{0}\right)\right),
$$

其中 $\sum_{m=0}^{n} P_{n}^{m}(\cos (m \lambda))=1$, 因此 $(3)$ 式简化为

$$
W(n)=(n+1) n^{2}\left|\frac{r_{0}}{a}\right|^{2[n-1]}\left|\frac{M_{r}}{a^{3}}\right| .
$$

由(4) 式可知, $W(n)$ 是 $M_{r}$ 和 $r_{0}$ 的函数, $W(n)$ 随 $M_{r}$ 和 $r_{0}$ 的减小而减小, 所以在地表观 测到的磁场能谱变化包含 $M_{r}$ 和 $r_{0}$ 两方面的影响. $W(n)$ 的减小既可以由 $M_{r}$ 衰减引起, 也可 以由偶极子向地心移动引起. 当 $n \geqslant 2$ 时, $W(n)$ 近似为一直线, $M_{r}$ 只影响这一拟合直线的截 距, 而 $r_{0}$ 则与其斜率相关:

$$
K=2 \lg \left(r_{0} / a\right) .
$$


$2 \boldsymbol{r}_{\mathbf{0}}$ 的长期变化对地球磁场能谱的影响

地球磁场能谱在空间的分布为

$$
W_{c}(n)=\left|\frac{a}{r}\right|^{2 n+2}(n+1) \sum_{m=0}^{n}\left(g_{n}^{m^{2}}+h_{n}^{m^{2}}\right),
$$

(6) 式中 $r$ 为参考面到地心的距离.

Meyer ${ }^{[11]}$ 把 1965 1985 年的地球磁场能谱归一到同一等效磁源, $r_{0}=3324 \mathrm{~km}$. 这与 Glatzmaier 等人 ${ }^{[13]}$ 估测的地核半径基本一致. 结果表明 $W_{c}(1)$ 线性递减, 而 $W_{c}(2)$ 和 $W_{c}(3)$ 几 乎以同一速率递增, 补偿了偶极子场的衰减, 总能量保持守恒. 即 $W_{c}(1)$ 的能量向 $W_{c}(2)$ 和 $W_{c}(3)$ 传递.

事实上, 地球磁场并非真的由放在 $r_{0}$ 的偶极子场产生, 从物理角度考虑只是一种等效的 概念. 在对数坐标下, 地球磁场的能谱在高阶项服从直线分布, 从统计意义上可以得出等效磁 源深度 $r_{0}$. 由图 1 可知, 地球磁场等效磁源深度 $r_{0}$ 并非一个常量. 1900 1940 年 $r_{0}$ 稳定递 减; 1940 1950 年 $r_{0}$ 阶跃性变化可能与某种扰动相关; 1950 1975 年 $r_{0}$ 呈现递减趋势; 1975 1985 年稳定递增; 1985 1995 年 $r_{0}$ 则基本保持不变.

为了分清能谱变化到底是由, 还是由 $M_{r}$ 引起. 不同于 Meyer 的处理方法, 本文把不同年 份能谱的 $W(1)$ 下延到各自的等效源深度. 结果表明(图 2), 归一到不同年代相应 $r_{0}$ 的曲线, 偶极子场的能量 1900 1960 年间基本保持不变, 1960 1970 年略微上升, 1970 年为转折点, 曲 线呈下降趋势. 说明 1900 1960 年间偶极子磁矩没有衰减. 非偶极子场能量则逐年增加, 暗 示非偶极子的磁矩递增. 地球磁场的总能量递增, 与地表显示的结果正好相反. 1900 1960 年间 $W_{c}(1)$ 基本保持水平, 这表明这一时期在地表 $W(1)$ 的变化是由等效源深度向地心移动 引起, 而并非是由偶极子场磁矩衰减引起.

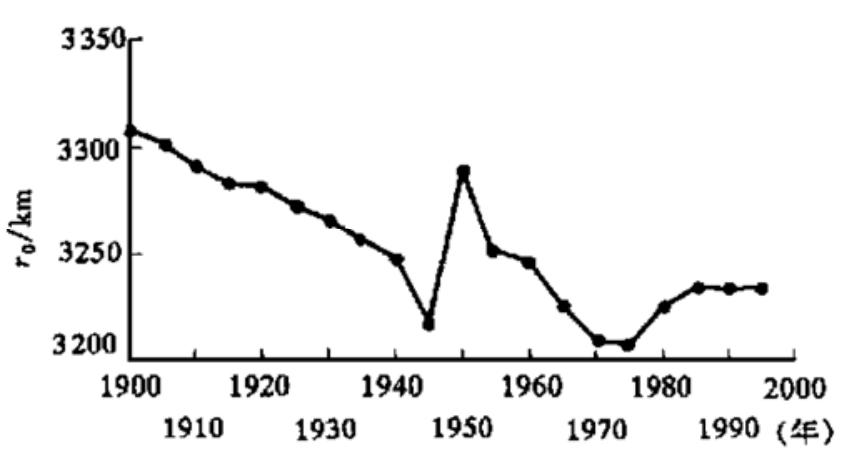

图 1 地球磁场等效磁源深度 $r_{0} 1900$ 年 以来长期变化图

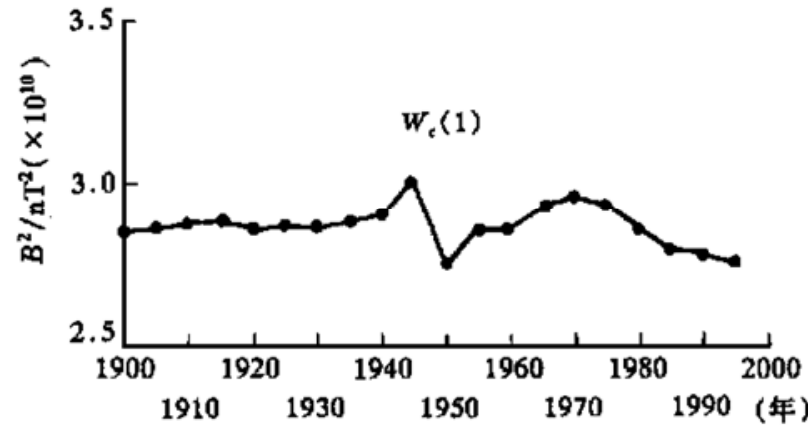

图 2 把每一年的地球磁场能谱归一到相应 $r_{0}$ 后, $W(1)$ 随时间变化曲线图

除去 1940 1950 年的扰动, $r_{0}$ 的变化明显在 1975 及 1985 年出现拐点. 这一变化规律与 1969 及 1979 年左右发生的地磁地磁阶跃有 $5 \mathrm{a}$ 左右的时间差, 这表明 $r_{0}$ 的长期变化与地磁阶 跃密切相关. 而地磁阶跃的形成又与日长变化相联系. 可见 $r_{0}$ 和地磁阶跃以及日长的变化在 成因上相互关联.

$3 \quad r_{0}$ 和地磁阶跃及日长变化的相互关系

欧洲一些地磁台站的观测资料去除外源场的影响后, 地磁场东向分量对时间的导数呈 V 
字形变化 ${ }^{[1,14]}$ (图 2), 这一变化规律具有全球型特征 ${ }^{[15,16]}$. V 字形的尖端称为地磁阶跃, 代 表地磁场长期变化中的加速阶跃. 发生在 1969 1970 及 1978 年的地磁阶跃已经被广泛研 究 ${ }^{[15,17,18]}$. 从地球磁场与外核流体耦合角度分析, 地磁场长期变化可由外核顶端流体运动对

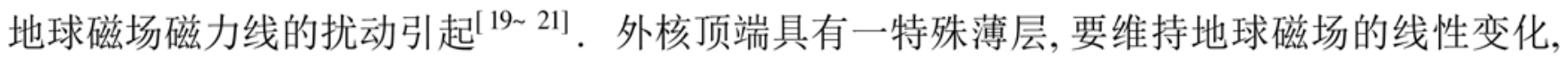
这一薄层的厚度必须大于 $75 \mathrm{~km}^{[22]}$.

对日长和地球磁场时间尺度为 $10 \mathrm{a}$ 的变化研究表明, 两者的变化趋势极其相近 ${ }^{[23,24]}$, 这 表明日长变化和地球磁场变化相关. Yoshida 等人 ${ }^{[25]}$ 认为地球磁场变化中的扇形成分由日长 变化引起. 当地幔的旋转速率变化时, 地幔底部会对核幔边界 (CMB) 产生冲击, 引起外核流体 的变化. 进而, 外核流体的变化与环型磁场耦合产生极型场. 外核流体运动速率与地球磁场 的时间导数成正比, 因此, 地球磁场 $\mathrm{V}$ 型变化可能由 $\mathrm{V}$ 型变化的流体与环型磁场耦合引起.

根据 Courtillot ${ }^{[1]}$ 的研究结果, 可以用核、幔耦合模型来解释日长和地球磁场的长期变化, 并且日长的变化滞后于地球磁场变化 10 15 a. 另一方面, 地震学和矿物物理学研究结果表 明, 核、幔界面是一个热学、力学、化学以及电磁学相互作用很复杂的区域. 地核中处于熔融态 的铁可能与地幔中的硅酸盐反应, 其结果形成物质结构和物理化学特征都极为复杂的 $\mathrm{D}^{\prime \prime}$

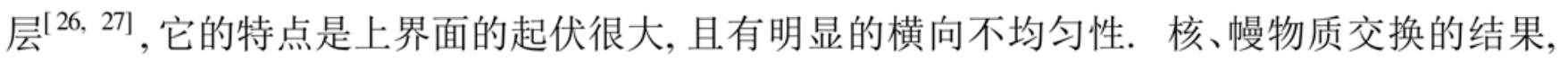
不但在 CMB 以上形成 $\mathrm{D}^{\prime \prime}$ 层, 而且还在 CMB 以下外核顶部形成一特殊薄层. 这样, 下地幔、外 核顶部特殊薄层及剩余的外核流体构成一个 3 层模型. 虽然相比较于地核, 地幔的电导率很 低, 但它仍具有传导性, 尤其下地幔. 核、幔间的电磁耦合不能忽略, 但是由于对地幔电导率及 $\mathrm{D}^{\prime \prime}$ 层的研究还欠深入, 这种核、幔电磁耦合还不能用来确定日长的变化. 另一种耦合是形态耦 合, 由于 CMB 的起伏, 流体的环型运动会对地幔产生较大的压力矩, 足以引起日长的变化. 径 向物质的交换对外核施加了一个力矩, 使其减速; CMB 的电磁耦合和形态耦合进而使地幔减 速. 地幔旋转速率的改变会反映在日长的变化之中.

以上表明, 下地幔底部对 CMB 的影响, 使得外核流体运动方式的改变. Jault ${ }^{[22]}$ 采用二维 双层流体模型探讨磁场与流体耦合的关系. $z=R-r, z$ 轴指向地心, $R$ 和 $r$ 分别为地球半径 和球心到任一点的半径, 特殊薄层厚度为 $\Delta, \xi=(R-r) / \Delta=Z / \Delta$ 流体模型为

$$
\begin{aligned}
& \bullet=\left(\boldsymbol{\bullet}^{0}(z, x), \boldsymbol{\bullet}^{\Delta}(z, x)\right) e^{-\varphi}, \\
& v_{x}^{0}(z, x)=v_{0} \exp [-K(z-\Delta)] \sin K x, \\
& v_{r}^{0}(z, x)=v_{0} \exp [-K(z-\Delta)] \cos K x, \\
& v_{x}^{\Delta}(\xi x)=-\frac{v_{0}}{K \Delta} \sin K x, \\
& v_{r}^{\Delta}(\xi x)=v_{0} \xi \cos K x .
\end{aligned}
$$

磁流体与主磁场耦合产生磁场的振幅表达式为

$$
F(t)=B K \gamma t B_{\varepsilon}(t / \Theta),
$$

其中 $\varepsilon=2 \pi \Delta L, L=1.5 \times 10^{6} \mathrm{~m}, B$ 为主磁场的振幅, $\gamma \approx \pi^{1} \times 10^{-12} \mathrm{~m}^{-1} \mathrm{~s}^{-2}, \Theta=\left(\mathrm{K}^{2}\right)^{-1}$, $\eta=2 \mathrm{~m}^{2} \cdot \mathrm{s}^{-1}$.

假设由于下地幔对 CMB 的冲击, 使外核流体向地心产生附加运动, 则流体的速度 ((7) 式) 向地心平移, 其结果使得特殊层的厚度 增大. 由公式(8) 可知, 特殊薄层厚度 $\Delta$ 增大, 
$B_{\varepsilon}(t / \Theta)$ 的值减小, 从而耦合磁场的振幅减小. 从统计意义上讲, $r_{0}$ 代表产生地球磁场源的平 均深度. 外核流体运动向地心方向迁移, 就相当于 $r_{0}$ 向地心迁移, 反之亦然. 可见 $r_{0}$ 的变化 是核幔耦合, 使外核流体运动方式发生改变的结果.

对于这样的一种 3 层模型, 地幔、外核顶部及外核其余流体的旋转速率与 2 个常量有关, 其一小于 $1 \mathrm{a}$, 另一个与核幔之间电导率 $\sigma_{c} / \sigma_{m}$ 有关, 如果日长滞后于磁场变化 $10 \sim 15 \mathrm{a}$, 则 $\sigma_{c} / \sigma_{m}$ 介于 500 1000 之间, 这与目前估测的值一致 ${ }^{[1]}$, 证实了这种 3 层模型的合理性.

\section{3 结论}

（1）地球表面观测的磁场的能谱在高阶项, 近似为一直线, 斜率与偶极子的深度相关;

(2) 地球磁场等效磁源深度 $\left(r_{0}\right)$ 并非一个常量, 它的变化规律与地磁阶越及日长变化趋 势一致. 核、幔耦合不仅能够引起地磁阶跃及日长的变化, 而且还能引起 $r_{0}$ 的长期变化;

(3) 1900 年以来, 其等效磁源的 $r_{0}$ 有向地心靠拢的趋势. 地球磁场偶极子能量的衰减是 由 $r_{0}$ 的变化引起, 其磁矩基本保持不变, 而非偶极子场的磁矩逐年递增.

致谢 对 Charles Barton 博士及徐元芳副研究员提供了 1990 年以来的地球磁场球谐系数, 作者深表感谢. 本 工作为国家杰出青年科学基金( 批准号: 49425405) 和中国科学院百人计划项目资助.

\section{参 考 文 献}

1 Courtillot V, Le Mouel J L. Geomagnetic secular variation impulses. Nature, 1984, 311: 709 716

2 Gubbins D. Finding core motions from magnetic observations. Phil Trans R Soc Lond Soc, 1982. 73: 247 254

3 Voorihies C V, Backus G. Steady flows at the top of the Earth's core from geomagnetic midels: the steady motions theorem. Geophys Astrophys Fluid Dyn, 1985, 32: 163 173

4 Bloxham J, Jackson A. Time dependent mapping of the magneic field at the coremantle boundary. J Geophys Res, 1992, 97 : 19537 $\sim 19563$

5 Davis R G, Whaler K A. The 1969 geomagnetic impulse and spirr up of the Earth's liquid core. Phys Earth Planet Inter, 1997, 103: 181 194

6 Courtillot V, Le Mou•l J L. Time variations of the Earth's magnetic field: from daily to secular. Ann rev Earth Planet Sci, 1988, 16: 389 476

7 Backus G E, Le Mouel J L. Application of mantle filter theory to the magnetic jerk of 1969. Geophys J R Astr Soc, 1983, 74: 713 746

8 Lowers F J. Mearr square values on sphere of spherical harmonic vector fields. J Geophys Res, 1966, 71: 2179

9 Langel R A, Estes R H. A geomagnetic field spectrum. Geophys Res Lett, 1982, 9: 250 253

10 Constable C, Parker R L. Statistical model for geomagnetic secular variation for the past 5 my. J Geophys Res, 1988, 93: 11569 11581

11 Benton E R, Alldredge L R. On the interpretation of the geomagnetic energy spectrum. Phys Earth Palnet Inter, 1987, 48: 265 278

12 Meyer J. Secular variation of magnetic mean energy density at the source-layer depth. Phys Earth Plant Inter, 1985, 39: 288 292

13 Glatamaier G A, Roberts P H. On the sounding of planetary interiors. Phys Earth Planet Inter, 1996, 98: 207 220

14 Gavoret J, Gibert D, Menvielle M, et al. Long-term variations of the external and internal components of the Earth's magnetic field. J Geophys Res, 1986, 91: 4787 4796

15 Mcleod M G. Signals and noise in magnetic observatory annual means: mantle conductivity and Jerks. J Geophys Res, 1992, 97: $17261 \sim 17290$

16 Stewart D N, Whaler K. Geomagnetic disturbances fields: an analysis of observatory monthly means. Geophys J Int, 1992, 108: 
$215 \sim 223$

17 Malin S R O, Hodder B M. Was the 1970 geomagnetic jerk of internal or external origin? Nature, 1982, 296: 726 728

18 Gubbins D. Geomagnetic field analysis. (E) secular variation consistent with a perfectly conducting core. Geophys J R Astron Soc, 1984, 77: 753 766

19 Bloxham J, Gubbins D. Thermal core-mantle interaction. Nature, 1987, 325: 511 513

20 Kohler M, Stevenson D J, Modeling core fluid motions and drift of magnetic field patterns at the CMB by use of topography obtained by seismic inversion. Geophys Res Lett, 1990, 17: 1473 1476

21 Jackson A, Bloxham J, Gubbins D. Time dependent flow at the core surface and conservation of angular momentum in the coupled core mantle system. Geophys Monogr Am Geophys Union, 1993, 72( IUGG vol. 1 2) : 97 107

Jault D, Le Mouel J L. Does secular variation involve motions in the deep core?. Physics Earth Plant Int, 1994, 82: 185 193

3 Langel R A, Kerridge D J, Barraclough D R, et al. Geomagnetic temporal change: 1903 1982, a spline respentation. J Geomagn Geoelectr, 1986, 38: 573 597

24 Le Mouel J L, Courtillot V, Jault D. Changes in Earth rotation rate. Nature, 1992, 355: $26 \sim 27$

25 Yoshida S, Hamano Y. Geomagnetic decadal variations caused by lengthr of day variation. Phys Earth Plan Inter, 1995, 91: 117 129

Knittle E, Jeanloz R. Earth's core-mantle boundary: results of experiments at high pressures and temperatures, Science, 1991, 251: 1438 1443

27 Lay T. Structure of the core_msntle transition zone. Eoc, 1989. 70

\title{
$\approx 37 \mathrm{kaBP}$ 大气中宇宙成大国位素含量 增加的古里雅冰芯证据
}

王宁练 姚檀栋 秦大河 L. G. Thompson ${ }^{(4)}$

\author{
E. Mosley-Thompson ${ }^{(4)}$ J. Cole-Dai ${ }^{(4)}$ \\ M. E. Davis ${ }^{(4)}$ P. N. $\operatorname{Lin}{ }^{(4)}$
}

( 中国科学院兰州冰川冻土研究所冰芯与寒区环境开放研究实验室, 兰州 730000 ;

(4) Byrd Polar Research Center, Ohio State University, USA)

摘要 证实了 $\approx 37 \mathrm{kaBP}$ 青藏高原古里雅冰芯记录中宇宙成因同位素 ${ }^{36} \mathrm{Cl}$ 含量峰值的存在, 并 认为该峰值是 ${ }^{36} \mathrm{Cl}$ 在大气中产生速率增加导致的, 而不是净积累速率变化影响的结果. 与其 他地区 ${ }^{10} \mathrm{Be}$ 及 ${ }^{36} \mathrm{Cl}$ 记录的对比, 指出宇宙成因同位素这一时期的峰值事件具有全球性, 可作为 冰芯等沉积物定年的时标. 发现这一峰值事件与气候变冷相伴出现.

\section{关键词 古里雅冰芯 宇宙成因同位素 ${ }^{36} \mathrm{Cl}$ 全球性事件 气候变化}

冰芯不但记录着地球气候环境变化的信息, 而且也记录着宇宙事件信息. 自从南极 Vostok 和 Dome $\mathrm{C}$ 两冰芯中发现宇宙成因同位素 ${ }^{10} \mathrm{Be}$ 浓度 $\left({ }^{10} \mathrm{Be}\right.$ 是宇宙射线与大气中 $\mathrm{N}$ 和 $\mathrm{O}$ 反应 的产物, 常吸附在气溶胶上, 并通过降水或干沉积降落至地表面, 其半衰期为 $\left.1.5 \times 10^{6} \mathrm{a}\right)$ 在 $\approx 35 \mathrm{kaBP}$ 出现峰值 ${ }^{[1,2]}$ 以来, 引起了气候环境研究和天文学研究 ${ }^{[3,4]}$ 的极大兴趣. 南极 Byrd 冰芯和格陵兰 Camp Century 冰芯中 ${ }^{10} \mathrm{Be}$ 浓度的分析结果, 进一步证实这一时期 ${ }^{10} \mathrm{Be}$ 浓度峰值的 